\title{
糖蛋白/糖肽分离富集中的化学
}

\author{
张丽霞杜秀芳曾盈* \\ (湖南师范大学化学化工学院化学生物学及中药分析教育部重点实验室 长沙 410081)
}

\begin{abstract}
摘要 糖蛋白在许多生命过程中有重要的作用. 糖蛋白/糖肽在复杂生物样品中的低丰度以及糖链结构的微观不均一 性使其分离富集成为糖蛋白质组学研究中的难点, 本文将糖蛋白/糖肽分离富集过程中使用的化学方法根据所应用的 化学反应或外源功能基团的种类分为: 肼化学方法, 氨化学方法, 硼酸化学法, $\beta$-消除米氏加成化学法; 并对各化学方 法的原理和优缺点进行了描述和讨论.
\end{abstract}

关键词 糖蛋白; 分离和富集; 糖蛋白组学; 化学

\section{Chemistry in Separation and Enrichment of Glycoproteins/Glycopeptides}

\author{
Zhang, Lixia \\ Du, Xiufang \\ Zeng, Ying*
}

(Key Laboratory of Chemical Biology and Traditional Chinese Medicine Research, Ministry of Education of China, College of Chemistry and Chemical Engeneering, Hunan Normal University, Changsha 410081)

\begin{abstract}
Glycoproteins play very important roles in many biological processes. The separation and enrichment of glycoproteins/glycopeptides is still considered a challenging task because of the low abundance and microheterogenity. This review introduces the chemistry method of separation and enrichment of glycoproteins and glycopeptides: hydrozide chemistry, amine chemistry, boronic acid chemistry, beta-elimination and Michael addition chemistry.
\end{abstract}

Keywords glycoprotein; separation and enrichment; glycoproteomics; chemistry

\section{1 引言}

蛋白质的糖基化修饰是最常见、最重要的翻译后修 饰之一, 对蛋白质的结构和功能有着很大影响. 蛋白质 的糖基化类型主要有两种: 一种是 $\mathrm{N}-$ 糖基化, 糖基片段 通过氮原子链接在天冬酰胺(Asn)的侧链上, 另一种为 $\mathrm{O}-$ 糖基化, 糖基片段通过氧原子链接在丝氨酸(Ser)或苏 氨酸( Thr)的侧链上, O-糖基化蛋白在碱性条件下易发生 $\beta$-消除反应. 糖基化蛋白在细胞间的识别、信息交流和 传递、病原对宿主的感染、胚胎的发生、癌症的发生和 转移等生物过程中起重要的作用 ${ }^{[1]}$. 在临床医学领域, 许多生物标志物及治疗的靶向目标是细胞外环境的糖 蛋白如癌症抗原 CA-125、AFP 和 CA19-9 等 ${ }^{[2]}$. 糖蛋白 的研究在生命科学和医学临床应用方面的重要意义, 吸 引了越来越多科学家的注意, 对糖蛋白的研究也得到飞 速的发展, 成为蛋白质研究的一个重要分支.

糖蛋白的研究主要包括以下几个方面: 糖蛋白的分 离富集; 糖基化位点及多肽序列的鉴定; 糖链的组成、 连接顺序及连接方式的鉴定; 糖基化修饰对蛋白质结 构、功能的影响; 对糖蛋白进行修饰改性及应用研究.
糖蛋白组学(Glycoproteomics)作为蛋白组学的一个分支, 是对生物体内糖蛋白的表达和变化进行规模化的识别、 检测和鉴定的研究, 是糖蛋白研究的关键内容. 糖蛋白 组学的研究对象是复杂的生物样品体系, 体系中蛋白质 的丰度范围大，高丰度非糖基化蛋白的存在对低丰度糖 蛋白有很大的干扰和影响, 而糖蛋白中糖链结构的微观 不均一性，更使糖蛋白的分离富集复杂化 ${ }^{[3]}$.

近年来利用糖蛋白的特殊物理化学性质, 在糖蛋白 分离富集方法方面的研究方面取得了很大的进步，也促 进了糖蛋白研究的发展. 糖蛋白/糖肽可以通过非化学

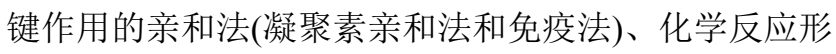
成化学键的化学方法、利用分子体积大小的尺寸排阻 法、利用糖基结构的亲水性的亲水色谱法或多种方法联 合使用等方法进行分离富集.

亲和法分离富集糖蛋白糖肽包括凝聚素法和免疫 法，利用糖蛋白中糖基片段的不同结构与不同凝聚素的 特异性作用, 或利用抗原抗体结合的专一性通过具有特 异性的单克隆抗体对相应的糖蛋白或糖肽进行分析. 凝 集素亲合法技术相对成熟, 可根据研究目标选择使用一 种或多种凝集素进行富集 ${ }^{[4 \sim 7]}$, 但凝集素本身的特性使

* E-mail: yingzeng@hunnu.edu.cn; Tel.: 0731-88872532

Received September 5, 2015; published November 12, 2015.

Project supported by the National Natural Science Fund of China (No. 21205038) and the Natural Science Foundation of Hunan Province, China (No. 13JJ2022).

项目受国家自然科学基金青年项目(No. 21205038)和湖南自然科学基金重点项目(No. 13JJ2022)资助. 
该方法不具备普适性, 特定的凝集素只能和对应一种或 多种类型的糖链发生相互作用, 且作用较弱. 免疫法灵 敏度高, 特异性好, 富集效率高, 但是抗体价格昂贵, 且操作复杂. 免疫法在富集和鉴定 O-乙酰氨基葡萄糖 基化的糖蛋白或糖肽中应用较多 ${ }^{[8,9]}$. 该方法特异性好, 富集效率高, 但抗体种类较少且价格昂贵, 并且操作复 杂，使该方法的应用有诸多限制。

糖链因其多羟基结构具有较强的亲水性, 许多亲水 性的层析介质都可以用来富集糖蛋白/糖肽 ${ }^{[10 \sim 12]}$, 但由 于连接糖链的肽段在疏水性上存在的很大的差异, 运用 亲水色谱法对糖肽或糖蛋白进行富集时可能会存在相 当大的非特异性吸附, 导致不能有效区分不同类型的糖 肽, 或未能彻底除去少量非糖基化蛋白质, 对后续研究 的准确性产生很大的影响.

糖与肽链间的链接方式以及糖环上不同于其它生 物分子的特殊邻位多羟基结构, 是利用化学方法进行糖 蛋白/糖肽分离富集的结构基础. 本文简要归纳总结了 利用化学反应形成化学键对糖蛋白/糖肽进行分离富集 的方法.

\section{2 分离富集中的化学方法}

糖链结构是糖蛋白/糖肽的分离富集的化学方法的 结构基础. 根据化学方法中的反应或外源功能基团的种 类将其分为以下几类: 肼化学方法, 氨化学方法, 嗍酸 化学法, $\beta$-消除米氏加成化学法.

\section{1 肼化学反应法}

肼化学反应法中的外源官能团是肼基团, 利用糖蛋 白/糖肽中糖基片段的多羟基结构一一邻二醇结构被氧 化后得到醛基, 醛基再与固相载体上的肼基官能团生成 腙, 从而进行分离和富集. 整个过程包括三步(图 1): (1) 醛基的生成, 利用高碘酸或高碘酸盐把糖链上的邻二羟 基氧化成醛; (2)偶联, 氧化形成的醛基和固相载体上的 肼基团反应形成腙, 将糖蛋白富集在固相载体上; (3)糖 基化蛋白或肽的释放.

肼化学方法中的外源性官能团也可以是苯肼或酰 肼 ${ }^{[13]}$; 通过改变氧化反应的条件可以选择性氧化唾液 酸的甘油侧链或甘露糖片段的顺式邻二醇, 或葡萄糖片 段的反式邻二醇. 糖蛋白/糖肽的释放方法也根据糖蛋 白/糖肽的类型而各异. 如用内切糖苷酶如 PNGase F 切 割 $\mathrm{N}$-糖基化蛋白或糖肽, 释放出去糖基化的肽; 用酸水 解链接的唾液酸可释放去唾液酸化的糖蛋白/糖肽; 使 用差弪胺通过水解切断腙键等方法, 将糖肽从固相载体释 放出来(图 2). Zhang 等 ${ }^{[14]}$ 于 2003 年首次使用此方法, 氧 化糖环上的顺式邻二醇, 通过肼与氧化生成的醛基反应 生成腙, 将人血清中的糖蛋白富集到树脂上, 再利用 PNGase $\mathrm{F}$ 将 $\mathrm{N}$-糖基化肽中的肽释放出来(图 2A). Klement 于 2009 年通过改变反应温度和氧化剂高碘酸
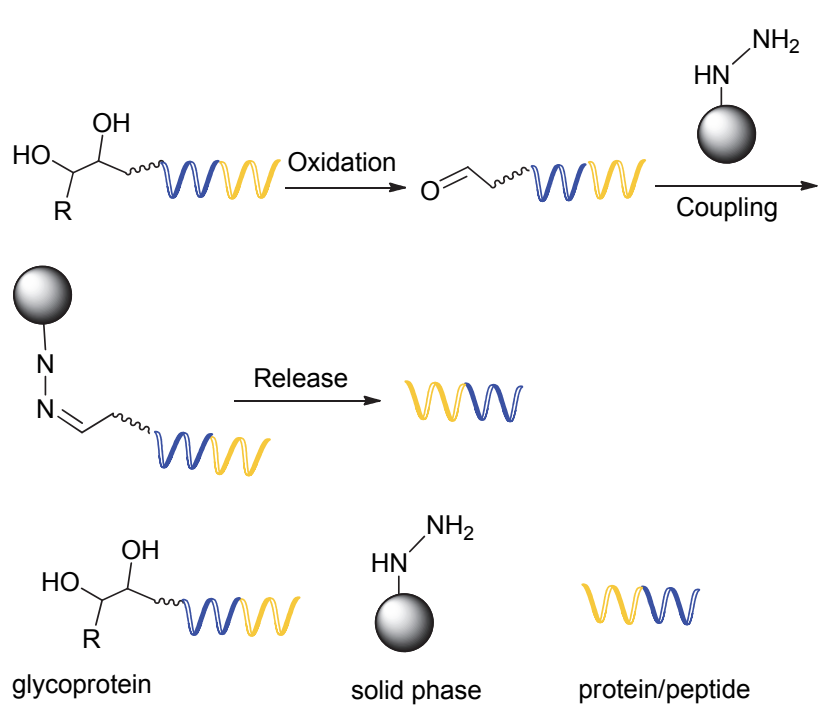

图 1 肼化学分离富集方法示意图

Figure 1 Illustration of hydrazide chemistry in separation and enrichment of glycoprotein/glycopeptide

浓度将 $O$-乙酰氨基葡萄糖基化蛋白糖环上的反式二醇 (氨基葡萄糖 3,4 位)进行氧化开环生成醛基, 开环后的 糖肽与酰肼树脂反应并结合到固相上，从而实现分离富 集, 反应后的树脂经羟胺处理发生腙键断裂, 释放出利 于质谱分析的开环糖肽肟衍生物 ${ }^{[15]}$ (图 2B). Larson 等 ${ }^{[16]}$ 通过低温度和低氧化剂浓度选择性地将唾液酸的甘油 侧链氧化生成醛基, 再通过含肼的固相载体对唾液酸化 的糖蛋白进行富集, 利用酸水解唾液酸的糖苷键, 释放 出去唾液酸化的糖肽/糖蛋白(图 2C).

肼化学反应法分离富集糖蛋白/糖肽的主要优势在 于肼化学反应法的反应专一性高且无位点偏向性, 有助 于糖基化位点的确认, 并且腙非常稳定, 可使用剧烈的 清洗方法, 且清洗过程蛋白损失较少. 但肼化学反应法 分离富集糖蛋白/糖肽需要氧化糖链, 且肼对糖链有水 解和脱乙酰的作用, 糖链结构的完整性会受到破坏; 富 集的糖蛋白/糖肽没有无偏向无选择性的释放方法, 如 PNGase $\mathrm{F}$ 在释放去糖基化肽链过程中不会释放含岩藻 糖的糖肽从而丢失糖链信息. 此外, 肼化学反应法属于 多步骤化学反应, 条件不易控制, 且操作较复杂.

\section{2 氨化学反应法}

氨化学反应法和肼化学反应法过程类似, 其外源官 能团是氨基, 邻二醇结构氧化得到的醛基与固相载体上 的氨基官能团生成碳氮双键或进一步反应，从而进行分 离和富集. 分离和富集的过程也分为氧化、偶联和释放 三步. 其中糖蛋白的氧化和释放过程和肼化学反应法类 似，但偶联过程中所运用到的化学反应不同.

氨化学反应法的关键步骤是羰基和氨基在酸性条 件下生成 schiff 碱. 因 schiff 碱的稳定性较差, 生成的 schiff 碱或通过 $\mathrm{NaBH}_{4}$ 还原形成稳定的 $\mathrm{C}-\mathrm{N}$ 单键; 或 通过与其它基团产生共轭，增强产物的稳定性. 


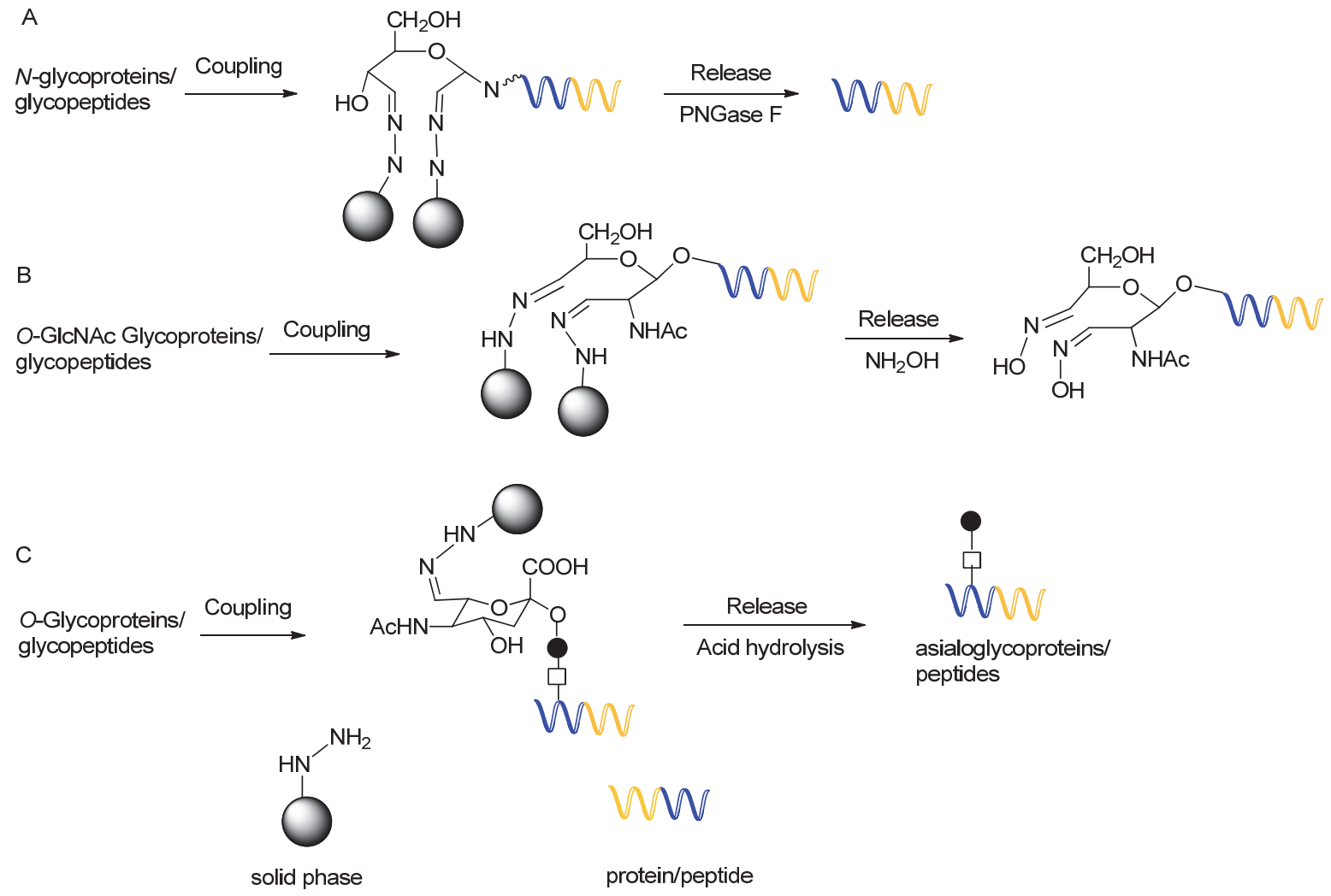

图 2 肼化学法链接糖蛋白/糖肽的释放示意图

Figure 2 Illustration of releasement of enriched glycoproteins/glycopeptides by hydrazide chemistry

$A$
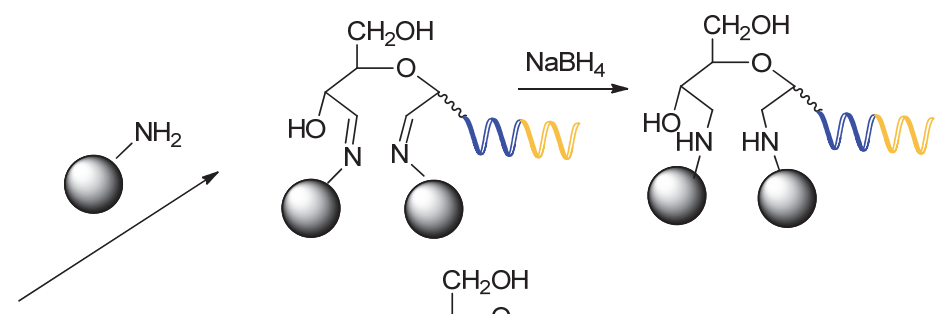

$B$
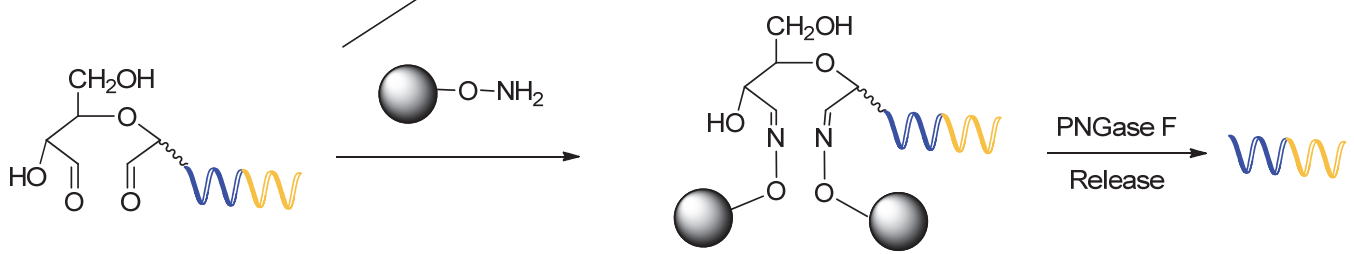

C
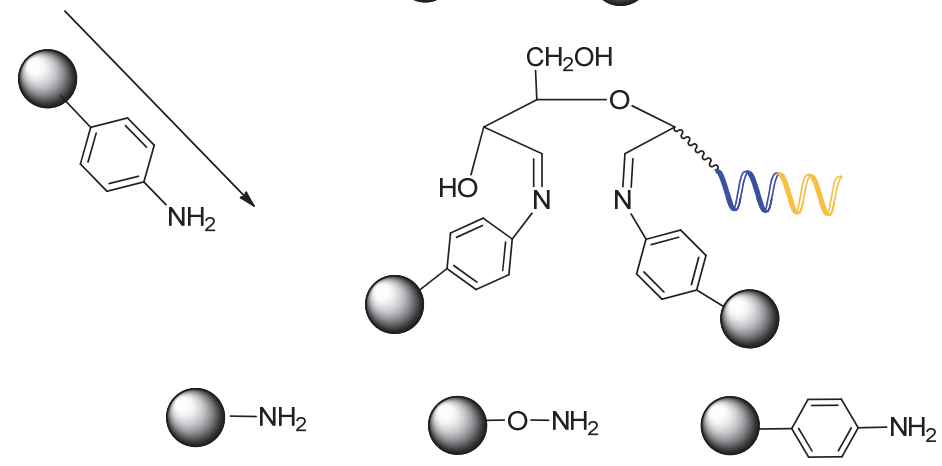

Different Amino Functionalized Solid Phase

图 3 氨化学法中所应用到的反应类型

Figure 3 Illustration of chemical reaction applied in amine chemistry 
陆豪杰研究组在氨化学反应法方面的研究最为广 泛及深入, 该研究组通过生成 schiff 碱再还原氨化的方 法, 用氨基功能化的磁性纳米粒子对 $\mathrm{N}$-连接的糖肽进 行分离富集, 并成功将核酸酶 B 从牛血清蛋白和核糖核 酸 $\mathrm{B}$ 的混合蛋白中分离出来(图3A $)^{[17]}$. 之后该研究组又 利用肜点击化学, 用氧氨基功能化的磁性纳米粒子高选 择性地对 $\mathrm{N}$-糖基化蛋白/肽进行分离富集, 并利用此方 法成功将人类大肠癌患者血清中的 $\mathrm{N}$-糖基化蛋白/肽分 离出来, 并对其进行了分析 ${ }^{[18]}$ (图 3B). 该研究组还通过 表面为苯胺修饰基团的纳米粒子通过苯胺环稳定化的 schiff 碱对 $\mathrm{N}$-链接的糖蛋白进行分离富集 ${ }^{[19]}$ (图 3C). 氨 化学法是近几年发展的新分离富集方法, 并逐步通过共 轭基团代替额外反应步骤增加产物的稳定性, 减少反应 步骤和简化操作. 氨化学法与肼化学法类似, 无偏向性, 但需要对糖链结构进行氧化, 糖链的完整性被破坏, 不 利于糖链结构的研究. 利用氨化学法对糖蛋白/糖肽大 规模普适性的分离富集的应用还需要进一步研究.

\section{3 嗍酸化学反应法}

嗍酸化学反应法同样利用糖蛋白/糖肽中糖基片段 上的顺式邻二醇结构来进行富集, 其最关键的相互作用 为硼酸配基与顺式邻二醇发生酯化反应. 在碱性条件 下, 硼酸与糖基片段中的顺式邻二醇发生酯化反应，生 成环状二酯, 将糖蛋白/糖肽富集到固相载体上; 在 $\mathrm{pH}$ 值小于 7 的条件下, 反应逆向进行, 环状二酯发生水解, 释放出糖蛋白或糖肽(图 4).

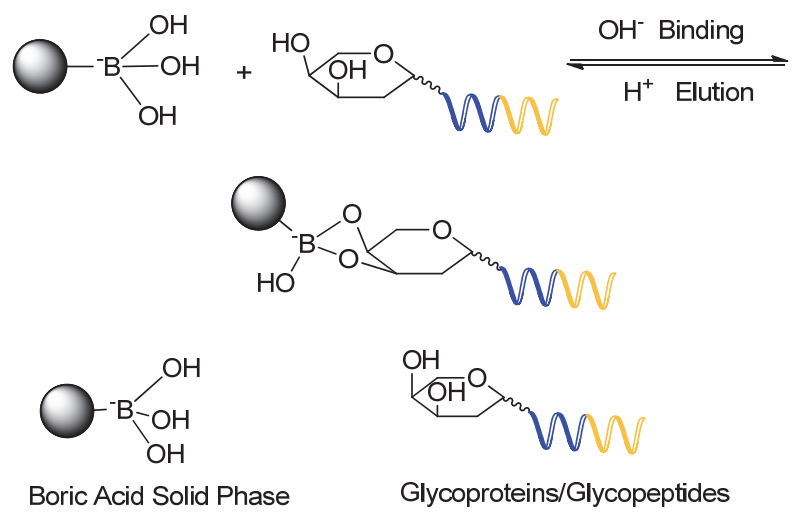

图 4 嗍酸化学法分离富集糖蛋白/糖肽的示意图

Figure 4 Illustration of separation and enrichment of glycoproteins/glycopeptides by boronic acid chemistry

嗍酸与糖蛋白/糖肽间的反应简单易行，硼酸已经 被修饰到各种固相载体上用于糖蛋白/糖肽的分离富集 中. Schulz 等 ${ }^{[20]}$ 通过嫁接在聚合物上的苯硼酸对人唾液 中的 $\mathrm{N}$-链接的糖蛋白进行了规模化的分离和分析, 发 现了 24 个新蛋白并确定了 67 个糖基化位点. 陆豪杰研 究组用苯嗍酸功能化的介孔纳米粒子选择性地富集糖 肽 $^{[21]}$. 张玉奎研究组制备了含嗍酸配体的不同聚合物 颗粒, 这些颗粒特异性的与糖蛋白结合, 能对糖蛋白进
行有效的分离富集 ${ }^{[22,23]}$. 张玉奎研究组制备的硼酸修饰 的纳米金粒子能从 HRP/BSA 的混合蛋白中高选择性地 富集 HRP, 该硼酸修饰的纳米粒成功规模化地鉴定识别 了人类血浆糖蛋白质的 160 个糖蛋白 ${ }^{[24]}$. 该研究组还制 备了不同含硼酸配体修饰的磁性粒子, 并研究了粒子对 糖蛋白进行分离富集，磁性粒子在 $\mathrm{pH}=7.4$ 的生理条件 下能对鸡蛋清中的糖蛋白进行分离富集 ${ }^{[25,26]}$. 目前用于 分离富集糖蛋白的嗍酸化聚合物和磁粒已商业化. Sparbier 等 ${ }^{[27]}$ 利用对硼酸功能化的磁性粒子对人血清中 的糖蛋白进行了规模化富集，并将该粒子的富集效果同 凝集素 ConA 和 WGA 的富集效果进行了比较, 结果表 明, 嗍酸富集法和凝集素富集法具有很好的互补性.

从化学反应原理上看, 嗍酸亲和色谱法分离富集糖 蛋白的优势在于嗍酸及其衍生物对糖蛋白/糖肽的捕获 无偏好性, 糖链中的顺式邻二醇结构都能与硼酸配体反 应生成酯而被富集. 由于硼酸捕获过程的酯化反应为可 逆反应，只需改变 $\mathrm{pH}$ 值就能将糖蛋白/糖肽释放下来, 过程中糖链结构不被破坏, 能获得完整的糖链信息, 也 不需要加入其他化合物, 减少了对后续实验的干扰. 但 糖蛋白/糖肽与硼酸配体除了能形成环状二酯的一级相 互作用外，还有氢键、疏水和络合等多种二级相互作用 会对这种富集方法产生影响，甚至这些二级相互作用可 能会上升为主要因素, 因此在具体的实验过程中应该根 据不同的硼酸配体、糖链结构及实验条件进行不断的探 索.

\section{$2.4 \beta$-消除米氏加成法}

由于 $O$-糖基化蛋白缺乏如 PNGase $\mathrm{F}$ 这样具有普适 性的酶, O-糖基化蛋白研究技术的发展受到一定的限制. 在对 $\mathrm{O}$-糖基化蛋白分离富集的研究中, $\beta$-消除米氏加成 法( $\beta$-Elemination/Michael addition reaction)的应用最为 广泛. $\beta$-消除米氏加成法利用 $\mathrm{O}$-链接的糖蛋白在碱性条 件下容易发生 $\beta$-消除反应形成双键，而反应生成的 $\alpha, \beta$ 不饱和双键易与亲核基团发生加成反应，在 $\mathrm{O}$-链接的 位点处修饰上如疏基等比较活泼的基团或生物素等有 特殊性质的结构(图 5), 并利用连接基团的特有性质来 选择性富集目标糖蛋白或糖肽.

Wells 等 ${ }^{[28]}$ 首次利用 O-糖蛋白在碱性条件下发生 $\beta$ 消除反应, 产生不饱和双键, 然后再加入二硫苏糖醇 (DTT)或生物素戊胺(BAP)进行米氏加成反应，然后通 过颈基亲和柱或生物素亲和柱对标记了的 O-糖肽进行 亲和富集及释放糖蛋白，对鼠脑中纯化的 Synapsin I 生 物样品进行了糖蛋白的富集和糖基化位点的分析. Cai 等 ${ }^{[29]}$ 使用氨水、甲基胺、二甲基胺三种亲核试剂对 $\beta$ 消除后的 O-糖肽进行米氏加成, 对比了三种试剂的反 应效果，并利用 LC-MS 测定了 $\mathrm{O}$-糖肽的糖基化位点. 但在此方法的反应条件下磷酸化蛋白或烷基化的半胱 氨酸也容易发生消除加成反应, 因此需要经过磷酸化酶 处理/或对照反应来消除干扰. 

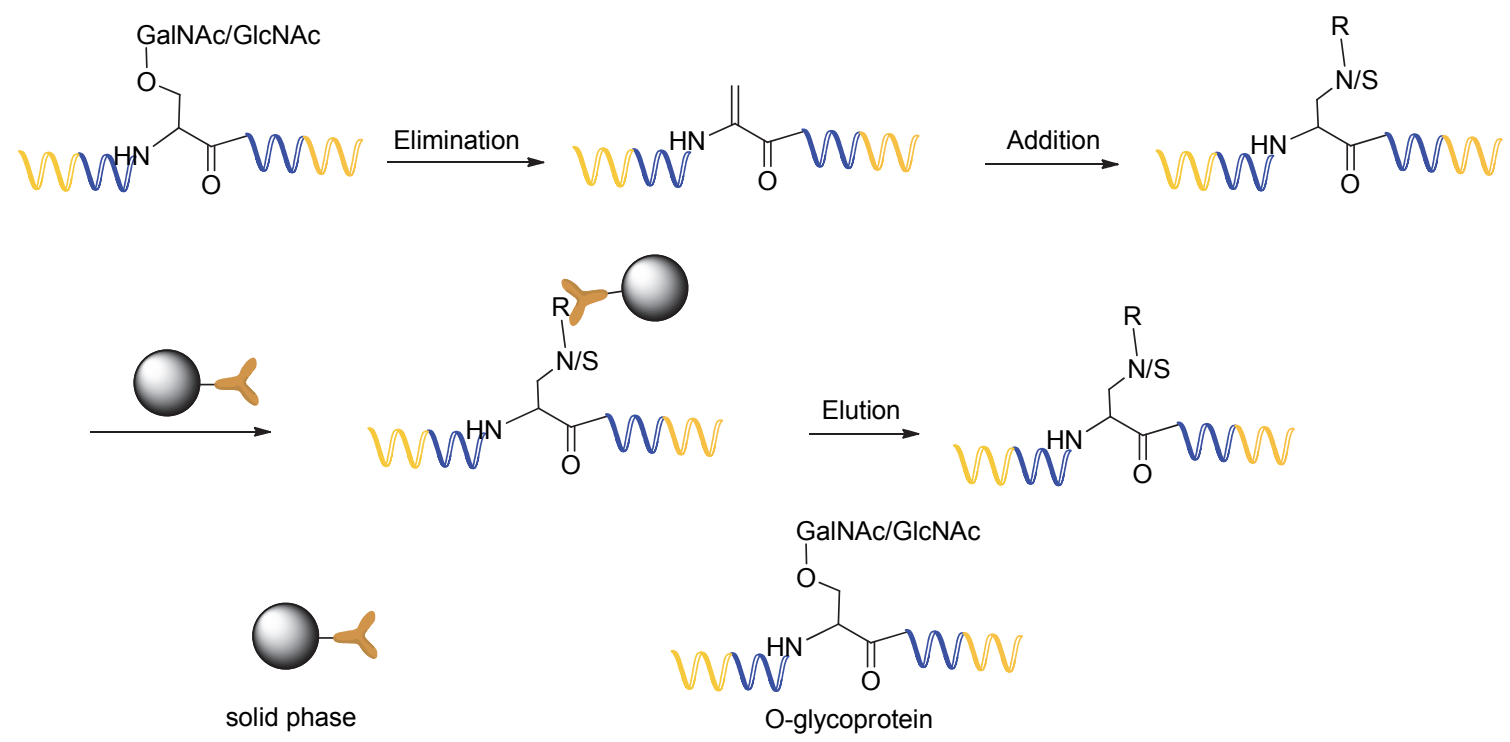

图 $5 \beta$-消除米氏加成法分离富集糖蛋白/糖肽的示意图

Figure 5 Illustration of separation and enrichment of glycoproteins/glycopeptides by $\beta$-Elimination/Michael addition reaction

\section{3 糖蛋白的分离和富集的策略}

利用化学反应法对糖蛋白的分离富集进行研究的 策略可分为 “top-down” 和 “bottom-up” 两种. 其中 “top-down” 策略属于蛋白水平的捕获, 先捕获蛋白质, 再用胰蛋白酶酶解固相载体上偶联的糖蛋白, 清洗去除 非糖基化肽后，释放去糖基化肽并且进行质谱鉴定; “bottom-up” 策略则是从肽段水平进行, 先将所用蛋白 进行酶解，再富集提取糖肽，并进行释放和质谱鉴定. “top-down”方法由于受到蛋白质的空间位阻和溶解性的 影响，其富集效率远不及“bottom-up”方法. Sun 等 ${ }^{[30]}$ 利 用散弹枪捕获法研究了 5 个标准糖蛋白/糖肽的富集实 验，验证了“bottom-up”方法确实比“top-down”方法具有 更高的效率, 其糖肽的富集选择性可达 $91 \%$. 目前, 各 种化学反应法分离和富集糖蛋白多采用“bottom-up”策 略.

\section{4 结束语}

通过糖蛋白/糖肽的特殊结构的化学性质对其进行 分离富集的方法多样, 但由于糖蛋白/糖肽中糖链结构 和性质的复杂性, 各化学分离富集方法各有优缺点. 嗍 酸法不破坏糖的结构也无偏好性, 能实现无痕分离富 集, 应用最为广泛, 却易与其他二级非化学键作用有干 扰; 而肼化学法和氨化学法都以邻二醇的氧化为化学基 础进行多步反应进行分离富集, 操作复杂, 易破坏糖链 的完整性; $\beta$-消除米氏加成法的反应条件易与磷酸化蛋 白等产生干扰. 目前还无法利用一种方法对全糖蛋白/ 糖肽进行高效、广谱富集. 因此在研究过程中研究者们 结合不同方法的优势同时使用多种富集方法对糖蛋白/ 糖肽进行分离富集和分析, 如凝集素亲和法和肼化学反

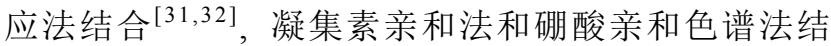
合 ${ }^{[33]}$, 亲水介质纤维素与连续凝集素亲和法结合使用 等 ${ }^{[34]}$. 多种方法联合使用能提高选择性和灵敏度, 提高 分离富集糖蛋白/糖肽的效率.

糖蛋白/糖肽的研究, 有助于全面细致地了解蛋白 质糖基化的结构与过程其生物学意义; 有助于从基因组 -蛋白组-糖组的宏观层面上分析生命中的现象并阐释 疾病发生的机制, 对有关疾病的早期诊断和治疗提供指 导. 近年来，随着科学技术的飞速发展，糖蛋白组学和 蛋白质组学都得到了极速的发展. 分离富集以及分析技 术的不断成熟与发展, 糖蛋白质组学将会在疾病蛋白质 组生物标志物的发现及药物靶点等临床应用中发挥极 其重要的作用. 现有的分离富集方法利用了糖蛋白/糖 肽中糖基片段的特殊结构和性质已经得到了广泛的应 用, 但由于糖蛋白中糖部分糖链结构的复杂性、微观不 均一性和多样性, 实现更全面、更低丰度糖基化蛋白质 的鉴定和分析仍需要不断开发新的分离富集方法和技 术，与质谱等先进技术结合，对糖蛋白/糖肽进行更深入 和广泛的研究.

\section{作者简介}

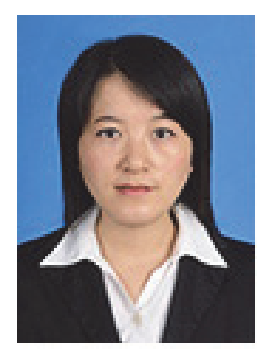

张丽霞, 女, 汉族, 湖南师范大学 2015 年毕业硕士生. 


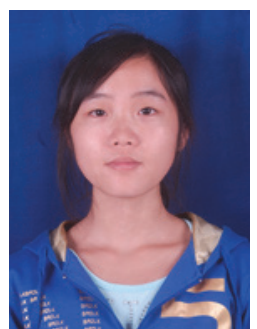

杜秀芳, 女, 汉族, 湖南师范大学在读研究生.

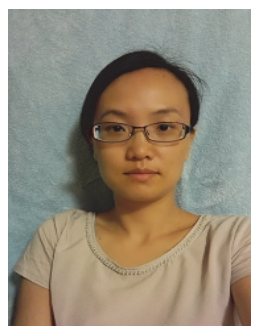

曾盈, 女, 湖南师范大学教授, 主要研究方向糖化学, 糖 化学生物学, 有机合成等.

\section{References}

[1] Varki, A.; Sharon, N. Essential of Glycobiology, 2nd ed., Eds.: Varki, A.; Cummings, R. D.; Esko, J. D.; Freeze, H. H.; Stanley, P.; Bertozzi, C. R.; Hart, G. W.; Etzler, M. E., Cold Spring Harbor Laboratory Press, New York, 2009, p. 1.

[2] Zhang, S. Z. Glycobiology and Glycoengineering, Tsinghua University Press, Beijing, 2002, p. 97. (张树政, 糖生物学和糖生物 工程, 清华大学出版社, 北京, 2002, p. 97).

[3] Kolli, V.; Schumacher, K. N.; Dodds, E. D. Bioanalysis 2015, 7, 113.

[4] Vosseller, K.; Trinidad, J. C.; Chalkley, R. J.; Specht, C. G.; Thalhammer, A.; Lynn, A. J.; Snedecor, J. O.; Guan, S.; Medzihradszky, K. F.; Maltby, D. A.; Schoepfer, R.; Burlingame, A. L. Mol. Cell. Proteomics 2006, 5, 923.

[5] Dai, Z.; Fan, J.; Liu, Y.; Zhou, J.; Bai, D.; Tan, C.; Guo, K.; Zhang, Y.; Zhao, Y.; Yang, P. Electrophoresis 2007, 28, 4382.

[6] Qiu, R.; Regnier, F. E. Anal. Chem. 2005, 77, 2802.

[7] Yang, Z.; Hancock, W. S. J. Chromatogr. A 2004, 1053, 79.

[8] Comer, F. I.; Vosseller, K.; Wells, L.; Accavitti, M. A.; Hart, G. W. Anal. Biochem. 2001, 293, 169.

[9] Ball, L. E.; Berkaw, M. N.; Buse, M. G. Mol. Cell. Proteomics 2006, 5,313 .

[10] Hagglund, P.; Bunkenborg, J.; Elortza, F.; Jensen, O. N.; Roepstorff, P. J. Proteome Res. 2004, 3, 556 .
[11] Zhao, J.; Simeone, D. M.; Heidt, D.; Anderson, M. A.; Lubman, D. M. J. Proteome Res. 2006, 5, 1126.

[12] Jiang, J.; Ying, W. T.; Qian, X. H. Chin. J. Anal. Chem. 2014, 42, 159. (江静, 应万涛, 钱小红, 分析化学, 2014, 42, 159).

[13] Liu, L.; Yu, M.; Zhang, Y.; Wang, C. C.; Lu, H. ACS Appl. Mater. Interfaces 2014, 6, 7823 .

[14] Zhang, H.; Li, X.; Martin, D. B.; Aebersold, R. Nat. Biotechnol. 2003, 21, 660 .

[15] Klement, E.; Lipinszki, Z.; Kupihar, Z.; Udvardy, A.; Medzihradszky, K. F. J. Proteome Res. 2010, 9, 2200.

[16] Nilsson, J.; Ruetschi, U.; Halim, A.; Hesse, C.; Carlsohn, E.; Brinkmalm, G.; Larson, G. Nat. Methods 2009, 6, 809.

[17] Zhang, Y.; Kuang, M.; Zhang, L.; Yang, P.; Lu, H. Anal. Chem. 2013, 85, 5535 .

[18] Zhang, Y.; Yu, M.; Zhang, C.; Ma, W.; Zhang, Y.; Wang, C.; Lu, H. Anal. Chem. 2014, 86, 7920.

[19] Zhang, Y.; Yu, M.; Zhang, C.; Wang, Y.; Di, Y.; Wang, C.; Lu, H. Chem. Commun. 2015, 51, 5982.

[20] Xu, Y.; Bailey, U. M.; Punyadeera, C.; Schulz, B. L. Rapid Commun. Mass Spectrom. 2014, 28, 471.

[21] Liu, L. T.; Zhang, Y.; Jiao, J.; Yang, P. Y.; Lu, H. J. Acta Chim. Sinica 2013, 71, 535. (刘丽婷, 张荣, 焦竞, 杨芃原, 陆豪杰, 化 学学报, 2013, 71, 535)

[22] Liu, J.; Qu, Y.; Yang, K.; Wu, Q.; Shan, Y.; Zhang, L.; Liang, Z.; Zhang, Y. ACS Appl. Mater. Interfaces 2014, 6, 2059.

[23] Liu, J.; Yang, K.; Qu, Y.; Li, S.; Wu, Q.; Liang, Z.; Zhang, L.; Zhang, Y. Chem. Commun. 2015, 51, 3896.

[24] Zhang, J.; Ni, Y. L.; Zheng, X. L. J. Sep. Sci. 2015, 38, 81.

[25] Zhang, X.; He, X.; Chen, L.; Zhang, Y. J. Mater. Chem. 2012, 22 , 16520 .

[26] Zhang, X.; He, X.; Chen, L.; Zhang, Y. J. Mater. Chem. B 2014, 2, 3254.

[27] Sparbier, K.; Wenzel, T.; Kostrzewa, M. J. Chromatogr. B, Anal. Technol. Biomed. Life Sci. 2006, 840, 29.

[28] Wells, L.; Vosseller, K.; Cole, R. N.; Cronshaw, J. M.; Matunis, M. J.; Hart, G. W. Mol. Cell. Proteomics 2002, 1, 791 .

[29] Zheng, Y.; Guo, Z.; Cai, Z. Talanta 2009, 78, 358.

[30] Sun, B.; Ranish, J. A.; Utleg, G. A.; White, T. J.; Yan, X.; Lin, B.; Hood, L. Mol. Cell. Proteomics 2007, 6, 141.

[31] Liu, T.; Qian, W. J.; Gritsenko, M. A.; Xiao, W.; Moldawer, L. L.; Kaushal, A.; Monroe, M. E.; Varnum, S. M.; Moore, R. J.; Purvine, O. S.; Maier, V. R.; Davis, W. R.; Tompkins, R. G.; Camp II, D. G.; Smith, R. D. Mol. Cell. Proteomics 2006, 5, 1899.

[32] Pan, S.; Wang, Y.; Quinn, J. F.; Peskind, E. R.; Waichunas, D.; Wimberger, J. T.; Jin, J.; Li, J. G.; Zhu, D.; Pan, C.; Zhang, J. J. Proteome Res. 2006, 5, 2769.

[33] Monzo, A.; Olajos, M.; Benedictis, L. D.; Rivera, Z.; Bonn, G. K.; Guttman, A. Anal. Bioanal. Chem. 2008, 392, 195.

[34] Kubota, K.; Sato, Y.; Suzuki, Y.; Goto-Inoue, N.; Toda, T.; Suzuki, M.; Hisanaga, S.; Suzuki, A.; Endo, T. Anal. Chem. 2008, 80, 3693. 